\title{
Sperm viability in the male accessory testes and female spermathecae of the bumblebee Bombus terrestris (Hymenoptera: Apidae)
}

\author{
Michael GREEFF and PAul SCHMID-HEMPEL \\ ETH Zürich, Institute of Integrative Biology (IBZ), Experimental Ecology, ETH-Zentrum CHN, Universitätsstrasse 16, \\ CH-8092 Zürich, Switzerland; e-mail: psh@env.ethz.ch
}

\begin{abstract}
Key words. Apidae, sperm storage, sperm viability, sperm competition, accessory testes, spermatheca, Bombus terrestris, bumblebees, colony cycle end
\end{abstract}

\begin{abstract}
In many species sperm competition selects for large ejaculates while females eliminate or disable a high percentage of the spermatozoa in their storage organs in order to control paternity. Therefore, sperm viability is an excellent measure of the reproductive success of both males and females. Here we assess the viability of spermatozoa in males, freshly mated queens and old queens at the end of a colony cycle of the monandrous bumblebee Bombus terrestris. We found that the accessory testes of males contained a significantly lower percentage of dead sperm (i.e. higher average viability) than the spermathecae of both freshly mated and old queens. In each case, however, the percentage of dead spermatozoa was very small. No differences could be detected between sperm viability in freshly mated and old queens. To test for the possible incompatibility of the sperm and the environment provided by female spermathecae males and females either from different geographic regions (north and south of the Alps) or the same region were mated. We did not find any differences between matings of individuals from within or between regions. The mechanism that causes the lower sperm viability in females remains unknown.
\end{abstract}

\section{INTRODUCTION}

In polyandrous species, males compete for parenthood of the offspring (Simmons, 2001) whereas females try to evade male manipulation and keep control over which male's sperm fertilize her eggs (cryptic female choice; Eberhard, 1996). As a result of this sexual conflict over fertilization males typically transfer large ejaculates of which females store but a small fraction; for example, this fraction is only $15 \%$ in Callosobruchus maculatus Fabricius (Eady, 1994). The larger part of the transferred sperm is either discarded by the female shortly after mating [e.g. carrion flies (Otronen \& Siva-Jothy, 1991)], degraded [bruchid beetle (Eady, 1994)], digested [flatworms (Michiels \& Bakovski, 2000)] or disabled within the female, for example, in the house fly (Degrugillier, 1985). Such sexually antagonistic coevolution can lead to a harsh environment within the female reproductive tract where only the sperm adapted to female manipulation can survive (Arnqvist et al., 2000). By comparing pairs of monandrous and a polyandrous species in seven closely related species Hunter \& Birkhead (2002) showed that in all cases the males of the polyandrous species had a higher percentage of living sperm in their sperm stores than their monandrous counterparts. This suggests that sexually antagonistic coevolution may generate rapid evolutionary divergence of reproductive traits and thereby facilitate the formation of new species, as shown by Arnqvist et al. (2000) in a meta-analysis of polyandrous and monandrous insect species [though another study did not show any connection between speciation and sexual selection (Gage et al., 2002)].
The mating biology of males of social insects has only received attention recently (Baer, 2003). In contrast to other well-studied insects like Drosophila (Chapman, 2001), most (but not all) of the social hymenoptera are monandrous (Boomsma \& Ratnieks, 1996) and therefore lack many of the typical features that evolved in polyandrous species. In addition, as many queens in this group often reproduce for a long time, e.g. some ants for up to several decades (Weber, 1972; Keller, 1998), they need both an efficient sperm storage system to maintain high sperm viability and very long lived sperm.

However, in the annual, monandrous bumblebee Bombus terrestris Linné there is evidence of paternal effects on the survival of the hibernating queens after artificial insemination (Korner \& Schmid-Hempel, 2003; Baer \& Schmid-Hempel, 2005). Since only pure sperm was transferred in these experiments, the effects may be ascribed to incompatibilities between males and queens. Such incompatibilities could stem from genomic factors (Zeh \& Zeh, 2003) or even more exotic processes like immunological incompatibilities. For example, after artificial insemination of $B$. terrestris Korner \& SchmidHempel (2003) found melanized spermathecae in some of the queens, which indicates that immune reactions may underlie these incompatibilities. Greeff \& SchmidHempel (2008) found melanized spermathecae in naturally mated, hibernating queens. These findings indicate the need to assess sperm viability in particular pairings of males and females, as variation in sperm viability within the female may provide a clue of the cause of these incompatibilities.

The male reproductive system of $B$. terrestris consists of two paired testes where sperm are produced until the 
males reach sexual maturity (Duchateau \& Marien, 1995). Afterwards, sperm are stored in the accessory testes until mating (Duvoisin et al., 1999). During mating males transfer not only sperm but also material that subsequently forms a mating plug inside the female's reproductive tract (Baer et al., 2000, 2001). The mating plug contains substances from the accessory glands that makes the queen refractory to re-mating (Sauter et al., 2001). Additionally, the plug also physically seals off the queen's reproductive tract (Baer et al., 2001). The mating plug disappears and its inhibitory effect on mating declines after a few days (Sauter et al., 2001). Male accessory gland products vary among different social bees but are known from all major groups and can modify the behaviour and physiology of females (Colonello \& Hartfelder, 2005). Similar functions for male gland products are recorded for the best-investigated insect, Drosophila melanogaster (Wolfner, 2002). During mating of bumblebees, sperm transferred by the male are released into the bursa copulatrix of the queen and, within approximately one hour, migrate through the spermathecal duct into the spermatheca where they are stored until fertilization. Fertilization of the eggs occurs much later when the female starts her own colony after hibernation (lasting July/August - March), thus, approximately eight months after mating (Schmid-Hempel \& SchmidHempel, 2000). Bumblebee males store enough sperm in their accessory testes to fully inseminate at least three females (Tasei et al., 1998).

Here we examined the viability of the spermatozoa in the accessory testes of males and in the spermathecae of queens after natural matings. Furthermore, the matings were arranged to occur either among bees originating from the same region or from two different geographic regions separated by the Alps to test for possible incompatibility. This approach was chosen, since genetic differentiation is recorded in other Bombus species (B. pascuorum; Widmer \& Schmid-Hempel, 1999) and crossinfection by parasites differs depending on geographic origin (Imhoof \& Schmid-Hempel, 1998). Since we found very high sperm viabilities both in males and freshly mated females an additional set of old queens taken from colonies in their final stage were used to assess sperm viability at the end of a colony cycle.

\section{METHODS}

Queens of B. terrestris collected in the field in the spring of 2003 in Ticino, south of the Alps, and around Zürich, north of the Alps, in Switzerland and allowed to establish colonies in the labortaory under standard conditions $\left(20-25^{\circ} \mathrm{C}, 60-70 \%\right.$ r.H., red light and food ad libitum). At the end of the colony cycle queens switch to laying sexuals; daughter (virgin) queens arise from fertilized eggs and males from haploid (i.e. unfertilized) eggs (Duchateau et al., 2004). Once a queen starts laying haploid eggs almost no fertilized eggs are laid anymore (Plowright $\&$ Plowright, 1990). The old queens from our colonies were dissected and sperm viabilities within their spermathecae were assessed. From the newly hatched sexuals, males (aged 7-16 days, from six different colonies) were mated with virgin queens (aged 5-10 days, from six other colonies). These dates corre- spond to the ages when the sexuals are most likely to mate (Duchateau \& Marien, 1995; Gretenkord, 1997).

Within $24 \mathrm{~h}$ of mating, males were dissected in buffer Kiev:M $(1.67 \mathrm{mM}$ glucose, $5.50 \mathrm{mM} \mathrm{KCl}, 2.50 \mathrm{mM} \mathrm{NaHCO} 3,8.26 \mathrm{mM}$ trisodium citrate; $\mathrm{pH} 8.8$ ). Each of the accessory testis was opened in a drop of $8 \mu \mathrm{l} \mathrm{Kiev:M.} \mathrm{The} \mathrm{non-sperm} \mathrm{tissue} \mathrm{was}$ removed and sperm viability was assessed with the LIVE/DEAD ${ }^{\mathrm{TM}}$ Sperm Viability Kit (L-7011, Molecular Probes), which consists of a membrane-permeant nucleic acid stain (SYBR14; diluted 1 : 50; emission max $516 \mathrm{~nm}$, green) and a dead-cell stain (propidium iodide; emission max. $617 \mathrm{~nm}$, red). For this purpose, $1.5 \mu 1 \mathrm{SYBR}(25 \mathrm{mM})$ were added to the sperm solution and gently mixed. Then, $1.5 \mu \mathrm{l}$ propidium iodide $(240 \mathrm{mM})$ was admixed and the sample on the slide covered with a cover slip $(21 \times 26 \mathrm{~mm})$. The accuracy of the staining method was checked using a dilution series of live and dead sperm. Immediately afterwards, pictures were taken using a microscope (Nikon Eclipse E600, UV-microscope, filter $420 \leq$ $\mathrm{EX} \leq 490, \mathrm{DM}=510, \mathrm{BA}=520$ ) of 10 different areas (subsamples) on the slide. From each sample the first 130 sperm, 13 in each of the 10 subsamples, were scored as red (dead), green (alive), or green-red (dying).

The young, freshly mated queens were kept in boxes with food ad libitum for five days and then dissected; the old queens were dissected on average 21 days after the first male emerged in their colonies. After dissection, the spermatheca was opened in a drop of $3.2 \mu 1 \mathrm{Kiev}: \mathrm{M}$ and the non-sperm tissue removed. The sperm were stained with $0.6 \mu \mathrm{l} \mathrm{SYBR}(25 \mathrm{mM})$ and $0.6 \mu 1$ propidium iodide $(240 \mathrm{mM})$, covered with a round cover slip $(\varnothing$ $7 \mathrm{~mm}$ ) and photographed under a microscope at ten different sites on the sample as described above. From each sample the first 100 sperm, 10 in each of the 10 subsamples, were scored as red (dead), green (alive), or green-red (dying).

In total, 106 young queens from 3 colonies from Ticino $(\mathrm{N}=$ $16,18,19)$ and 3 from Zürich $(N=17,18,19)$ were mated with 106 males from 3 different colonies from Ticino $(\mathrm{N}=16,18$, $18)$ and 3 from Zürich $(\mathrm{N}=18,18,18)$. Bees were never crossed with members of the same colony. On average, 2 to 5 pairs per patriline $\times$ matriline combination were mated. Old queens were taken from 6 colonies from Ticino and 24 from Zürich; each colony had only one old queen.

Data were analyzed with SPSS, v. 11 for Macintosh. The percentage values for the sperm were arcsin-transformed to normalize residuals as indicated by a Kolmogorov-Smirnov test. Sperm viabilities within the accessory testes and spermathecae were compared after classification into viable (green and greenred) and dead (red) sperm. The viable category is justified, as in a study on sperm survival in parasitoid wasps, Damiens et al. (2002) showed that green-red stained sperm are viable, and that dual-stained sperm is an artifact of the method. Here we consider the green-red sperm as viable. In order to compare the sperm viability within the accessory testis and spermathecae of the young queens a Wilcoxon paired test was performed on the percentages of dead sperm. For the analysis of sperm viability in each male, the means for the left and right accessory testis was used. The effects of matings between- and within-regions on sperm viability were analyzed using a nested ANOVA with two-tailed probabilities (same queens as used in the male-female comparison) using again the percentage of dead sperm. To normalize this data the means were calculated for each patriline $\times$ matriline combination, resulting in the 36 values used in the analysis ( 6 colonies from which males were taken and 6 colonies from which queens were taken). The nested ANOVA included the random factors, region of male origin and nested within it the colonies of the males, the region of queen origin and nested within it the colonies of the queens, the date of mat- 


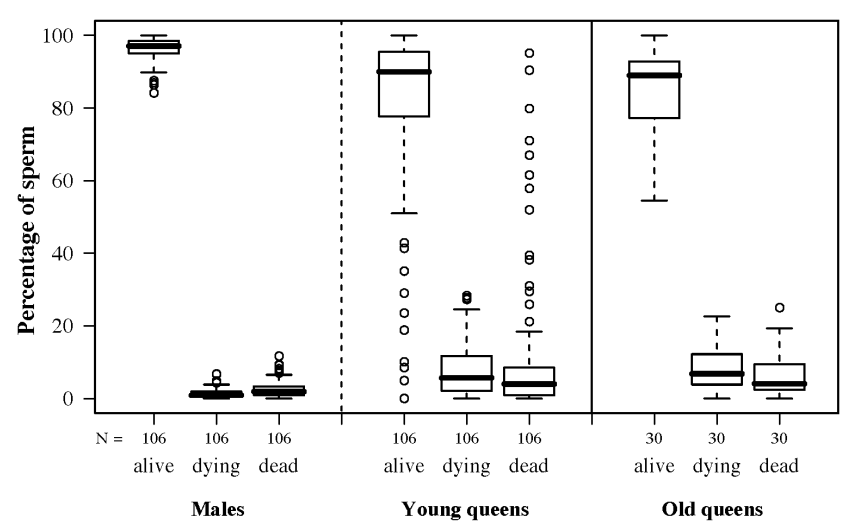

Fig. 1. Box plot of the percentages of live, dying and dead sperm in the bumblebee $B$. terrestris. Sperm were taken from the accessory testes of males (mean of right and left testis) immediately after mating, from the spermathecae of young queens five days after mating and from old queens at the end of the colony cycle (after the switch to laying unfertilized eggs). Males had a significantly higher percentage of viable (live and dying) and a significantly lower percentage of dead sperm $(\mathrm{p}<$ $0.001)$ than in the spermathecae of young queens. There were no significant differences in the sperm from the spermathecae of young and old queens $(p=0.291)$. For details of statistics, see text.

ing, as well as the interaction "male-region" $\times$ "queen-region". If matings between populations (i.e. between the regions Zürich and Ticino) differ in their compatibility from those within populations (i.e. within either region), a significant statistical interaction term is expected. Sperm viabilities within the spermathecae of old and young queens were analyzed with a Mann-Whitney U-test on the percentage of dead sperm.

\section{RESULTS}

The median percentage of live sperm in the accessory testes of males was $97.1 \%$ (interquartile range $95.0-98.5 \%$ ), $0.897 \%$ of dying sperm (interquartile range $0.361-1.92 \%$ ) and $1.92 \%$ of dead sperm (interquartile range $0.962-3.37 \%$ ) (Fig. 1). The median percentage of live sperm in the spermathecae of young queens was

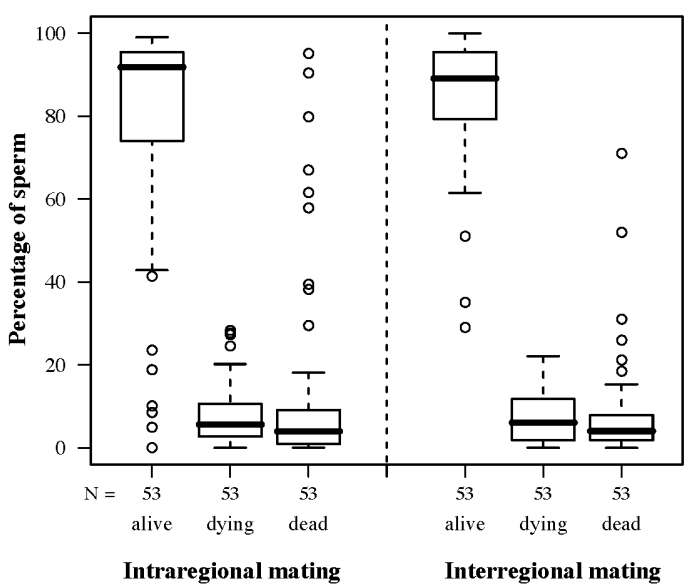

Fig. 2. Box plot of the percentages of live, dying and dead sperm within the spermathecae of young queens of the bumblebee $B$. terrestris five days after mating. 53 queens were mated with males from a region on the other side of the Alps (interregional) and 53 with males from their own region (intraregional). No significant differences were found between intraregional and interregional matings $(\mathrm{p}=0.312$, Table 1). Same data as in Fig. 1.

$90.0 \%$ (interquartile range $77.6-95.5 \%$ ), 5.69\% of dying sperm (interquartile range $2.11-11.8 \%$ ) and $4.02 \%$ of dead sperm (interquartile range $0.962-8.59 \%$ ). The median percentages in old queens was $89.0 \%$ live sperm (interquartile range 75.9-92.9\%), 6.82\% dying sperm (interquartile range $3.72-12.4 \%$ ) and $4.10 \%$ dead sperm (interquartile range 2.38-9.58\%) (Fig. 1).

The accessory testes of males contained significantly more viable (sum of live and dying) and significantly less dead sperm than the spermathecae of queens (Wilcoxon signed ranks test, $\mathrm{N}=106, \mathrm{Z}=-3.76, \mathrm{p}<0.001)$. However, there were no significant differences in the percentages of viable (percentage of live and dying sperm) or dead sperm in young and old queens (Mann-Whitney Test, $\mathrm{N}=136, \mathrm{P}=0.302$ ).

TABLE 1. General linear model of sperm survival within the spermathecae of young queens five days after mating.

\begin{tabular}{|c|c|c|c|c|}
\hline Source $^{1}$ & d.f. & MS & $\mathrm{F}$ & P-value \\
\hline Intercept & 1 & 7.38 & 0.088 & 0.77 \\
\hline error & 23 & 84.2 & & \\
\hline Male region & 1 & 69 & 21.4 & 0.994 \\
\hline error & 0.001 & 3.22 & & \\
\hline Male colony & 4 & 5.7 & 0.068 & 0.991 \\
\hline error & 23 & 84.1 & & \\
\hline Female region & 1 & 174 & 2.52 & 0.234 \\
\hline error & 2.37 & 69.1 & & \\
\hline Female colony & 4 & 53.7 & 0.639 & 0.64 \\
\hline error & 23 & 84.1 & & \\
\hline Date of mating & 1 & 3.29 & 0.039 & 0.845 \\
\hline error & 23 & 84.1 & & \\
\hline Male region*Female region & 1 & 89.9 & 1.07 & 0.312 \\
\hline error & 23 & 84.1 & & \\
\hline
\end{tabular}

${ }^{1}$ male region, female region, male colony, female colony as random effects; date mating as covariate; male colony nested within male region, female colony nested within female region. 
In the spermathecae of young queens (same queens as in the male-female comparison) after intra-regional matings, the median percentage was $91.8 \%$ live sperm (interquartile range $71.9-95.5 \%), 5.61 \%$ dying sperm (interquartile range $2.73-11.4 \%$ ) and $4.00 \%$ dead sperm (interquartile range 0.962-9.55\%). After inter-regional matings (same queens as in the male-female comparison), the median percentage in the spermathecae of young queens was $89.1 \%$ live sperm (interquartile range 78.7-95.7\%), 6.09\% dying sperm (interquartile range $1.82-11.9 \%$ ) and $4.04 \%$ dead sperm (interquartile range $1.39-8.07 \%$ ). Sperm viability within the spermathecae of young queens was tested using an ANOVA of the percentage of dead sperm. Contrary to expectation, the interaction male-region $\times$ queen-region, which should indicate the respective incompatibilities, was not significant $(\mathrm{p}=$ 0.312, Table 1; Fig. 2).

\section{DISCUSSION}

The percentage of dead sperm in the accessory testes of males was significantly lower than in the spermathecae of young queens, i.e. the viability of sperm was less within females. This result with those of other studies on sperm survival after mating, so far mostly done on nonhymenopteran insect species. In the polyandrous fly Scatophaga stercoraria L., for example, Bernasconi et al. (2002) not only found reduced sperm viability in females (in males: $\sim 86 \%$ alive; in females: $\sim 79 \%$ ), but also that an extract of the female's reproductive tract tissue did not result in a higher percentage of dead sperm in an in vitro test. In two polyandrous Photinus fireflies, sperm viability in male seminal vesicles was quite high (over $75 \%$ ) but dropped significantly inside the reproductive tract of females (Demary, 2005).

The only studies previously done on Hymenoptera point in the opposite direction (Damiens et al., 2002): in the polyandrous parasitoid Eupelmus orientalis Crawford, both male seminal vesicles and female spermathecae contain similar high percentages (ca. 30\%) of dead spermatozoa; in the polyandrous parasitoid Dinarmus basalis Rondani, males store sperm of which more than $40 \%$ is nonviable whereas in queens it is around $15 \%$. In $B$. terrestris, the long reproductive life span of the queens may account for the generally low percentage of dead sperm both in males and females. Hunter \& Birkhead (2002) measured the viability of spermatozoa in male $B$. terrestris and record a percentage of over $95 \%$ live spermatozoa. Why sperm-viability within the spermathecae of a monandrous species like $B$. terrestris is lower, as recorded here does not appear reasonable for a monandrous species.

However, sperm storage is costly in another social insect (leaf-cutter ants, Baer et al., 2006) in terms of reduced immune responsiveness. Therefore, bees might limit the nourishment provided to the sperm in order to fuel the immune system at the cost of letting sperm perish. In fact, Schoeters \& Billen (2000) report a high glycogen content in the cells lining the reproductive tract of females of $B$. terrestris and assume it serves as an energy source for the sperm. This might represent a small cost, since the number of sperm does not seem to be a limiting constraint. Out of the 40'000-50'000 spermatozoa stored within the spermatheca after copulation queens of $B$. terrestris use only a tiny fraction (Röseler, 1973). These large numbers furthermore allow some form of cryptic female choice even in a monandrous species like $B$. terrestris: indeed, the variation in sperm lengths is considerably smaller in the spermathecae of females than in the accessory testis of males (Baer et al., 2003). It is not known how sperm are selected within the female, nor is it clear why such selection occurs, since all sperm from the same male are genetically identical.

Mating incompatibilities between males and females are reported in other animals (Zeh \& Zeh, 2003) and could result in higher sperm mortality in certain malefemale genotype combinations in B. terrestris. At least in the honeybee, Apis mellifera L., spermatozoa from conspecific males remain motile for longer (after artificial insemination) than those from heterospecific males (Phiancharoen et al., 2004). Our data for B. terrestris did not show any differences between inter- vs. intra-regional matings (from both sides of the Alps), which we assumed would reveal mating incompatibilities. It is of course not known whether the Alps are a sufficient barrier to induce differences in compatibility, since European mainland populations of $B$. terrestris show virtually no genetic differentiation based on microsatellite variation (Estoup et al., 1996). On the other hand, cross-infection with parasites showed a difference in local adaptation in B. terrestris from the same Zürich (north of Alps) and Ticino (south) regions in $B$. terrestris (Imhoof \& SchmidHempel, 1998). It is still possible that genes coding for sexual traits behave differently from the microsatellites that were analyzed by Estoup et al. (1996) or those affecting host resistance, which diverged between regions north and south of the Alps.

The high viability of sperm within the spermathecae of old queens found in our study throws light on another very interesting question. Due to the haplo-diploid sex determination system workers are more related to sisters than to brothers whereas the mother queen of the colony is equally related to daughters and sons. The resultant conflict over optimal investment ratio was first described by Trivers \& Hare (1976) on the basis of Hamilton's kin selection theory (Hamilton, 1964a, b). Bumblebees have a highly male-biased population sex investment ratio (Bourke, 1997). Although in bumblebees the sex ratio is largely controlled by the queen (Duchateau et al., 2004), a limitation on the availability of viable sperm towards the end of the colony cycle could also explain the male bias (males originate from unfertilized eggs). However, as there was a high percentage of viable sperm in the spermathecae of old queens (after her switch to laying unfertilized eggs), the production of males appears to be a strategy of the queen rather than due to sperm limitation. Queens possess enough sperm to produce a colony because they do not contain significantly less sperm at the end of a colony cycle (Röseler, 1973). Rather than 
musing about why sperm die within the spermathecae of queens the question should be why do males transfer such large ejaculates even under monandry.

ACKNOWLEDGEMENTS. We thank Ch. Bressac for advice on staining and R. Schmid-Hempel for help with the rearing of bumblebee colonies. This study was supported by grants from the Swiss National Science Foundation (Nos 3100-066733, 3100A0-116057), ARC Discovery Grant (DP0209447) and CCES to PSH.

\section{REFERENCES}

Arnqvist G., Edvardsson M., Friberg U. \& Nilsson T. 2000: Sexual conflict promotes speciation in insects. Proc. Natl. Acad. Sci. USA 97: 10460-10464.

BAER B. 2003: Bumblebees as model organisms to study male sexual selection in social insects. Behav. Ecol. Sociobiol. 54: 521-533.

Baer B. \& Schmid-Hempel P. 2005: Sperm influences female hibernation success, survival and fitness in the bumble-bee Bombus terrestris. Proc. R. Soc. Lond. (B) 272: 319-323.

Baer B., Maile R., Schmid-Hempel P., Morgan E.D. \& Jones G.R. 2000: Chemistry of a mating plug in bumblebees. $J$. Chem. Ecol. 26: 1869-1875.

Baer B., Morgan E.D. \& Schmid-Hempel P. 2001: A nonspecific fatty acid within the bumblebee mating plug prevents females from remating. Proc. Natl. Acad. Sci. USA 98: 3926-3928.

Baer B., Schmid-Hempel P., Hoeg J.T. \& Boomsma J.J. 2003: Sperm length, sperm storage and mating system characteristics in bumblebees. Insectes Soc. 50: 101-108.

Baer B., Armitage S.A.O. \& Boomsma J.J. 2006: Sperm storage induces an immunity cost in ants. Nature 441: 872-875.

Bernasconi G., Hellriegel B., Heyland A. \& Ward P.I. 2002: Sperm survival in the female reproductive tract in the fly Scathophaga stercoraria (L.) J. Insect Physiol. 48: 197-203.

Boomsma J.J. \& RatnieKs F.L.W. 1996: Paternity in eusocial Hymenoptera. Philos. Trans. R. Soc. Lond. (B) 351: 947-975.

Bourke A.F.G. 1997: Sex ratios in bumble bees. Philos. Trans. R. Soc. Lond. (B) 352: 1921-1932.

Chapman T. 2001: Seminal fluid-mediated fitness traits in Drosophila. Heredity 87: 511-521.

Colonello N.A. \& Hartfelder K. 2005: She's my girl - male accessory gland products and their function in the reproductive biology of social bees. Apidologie 36: 231-244.

Damiens D., Bressac C., Brillard J.P. \& Chevrier C. 2002: Qualitative aspects of sperm stock in males and females from Eupelmus orientalis and Dinarmus basalis (Hymenoptera: Chalcidoidea) as revealed by dual fluorescence. Physiol. Entomol. 27: 97-102.

Degrugillier M.E. 1985: In vitro release of housefly Musca domestica L. (Diptera: Muscidae) acrosomal material after treatments with secretions of female accessory-gland and micropyle cap substance. Int. J. Insect Morphol. 14: 381-391.

Demary K.C. 2005: Sperm storage and viability in Photinus fireflies. J. Insect Physiol. 51: 837-841.

Duchateau M.J. \& Marien J. 1995: Sexual biology of haploid and diploid males in the bumble bee Bombus terrestris. Insectes Soc. 42: 255-266.

Duchateau M.J., Velthuis H.H.W. \& Boomsma J.J. 2004: Sex ratio variation in the bumblebee Bombus terrestris. Behav. Ecol. 15: 71-82.

Duvoisin N., Baer B. \& Schmid-Hempel P. 1999: Sperm transfer and male competition in a bumblebee. Anim. Behav. 58: $743-749$.
EADY P. 1994: Sperm transfer and storage in relation to sperm competition in Callosobruchus maculatus. Behav. Ecol. Sociobiol. 35: 123-129.

Eberhard W.G. 1996: Female Control: Sexual Selection by Cryptic Female Choice. Monographs in Behaviour and Ecology. Princeton University Press, Princeton, NJ, 472 pp.

Estoup A., Solignac M., Cornuet J.M., Goudet J. \& Scholl A. 1996: Genetic differentiation of continental and island populations of Bombus terrestris (Hymenoptera: Apidae) in Europe. Mol. Ecol. 5: 19-31.

Gage M.J.G., Parker G.A., Nylin S. \& Wiklund C. 2002: Sexual selection and speciation in mammals, butterflies and spiders. Proc. R. Soc. Lond. (B) 269: 2309-2316.

Greeff M. \& Schmid-Hempel P. 2008: Sperm reduces female longevity and increases melanization of the spermatheca in the bumblebee Bombus terrestris L. Insectes Soc. 55: 313-319.

GRETENKORD C. 1997: Laborzucht der Dunklen Erdhummel (Bombus terrestris L.) (Hymenoptera, Apidae) und toxikologische Untersuchungen unter Labor- und Halbfreilandbedingungen. Ph.D. thesis, University of Bonn, $356 \mathrm{pp}$.

HAMiLTON W.D. 1964a: Genetical evolution of social behaviour 1. J. Theor. Biol. 7: 1-16.

HAMiLton W.D. 1964b: Genetical evolution of social behaviour 2. J. Theor. Biol. 7: 17-52.

HunTER F.M. \& BiRKHEAD T.R. 2002: Sperm viability and sperm competition in insects. Curr. Biol. 12: 121-123.

Imноо B. \& Schmid-Hempel P. 1998: Patterns of local adaptation of a protozoan parasite to its bumblebee host. Oikos 82: $59-65$.

Keller L. 1998: Queen lifespan and colony characteristics in ants and termites. Insectes Soc. 45: 235-246.

Korner P. \& SChmid-Hempel P. 2003: Effects of sperm on female longevity in the bumble-bee Bombus terrestris $\mathrm{L}$. Proc. R. Soc. Lond. (B) 270: S227-S229.

Michiels N.K. \& BAKovsKi B. 2000: Sperm trading in a hermaphroditic flatworm: reluctant fathers and sexy mothers. Anim. Behav. 59: 319-325.

Otronen M. \& Siva-Jothy M.T. 1991: The effect of postcopulatory male-behavior on ejaculate distribution within the female sperm storage organs of the fly, Dryomyza anilis (Diptera, Dryomyzidae). Behav. Ecol. Sociobiol. 29: 33-37.

Phiancharoen M., Wongsiri S., Koeniger N. \& Koeniger G. 2004: Instrumental insemination of Apis mellifera queens with hetero- and conspecific spermatozoa results in different sperm survival. Apidologie 35: 503-511.

Plowright R.C. \& Plowright C.M.S. 1990: The laying of male eggs by bumble bee queens - an experimental reappraisal and a new hypothesis. Can. J. Zool. 68: 493-497.

Röseler P. 1973: Die Anzahl Spermien im Receptaculum Seminis von Hummelköniginnen (Hym., Apoidea, Bombinae). Apidologie 4: 267-274.

Sauter A., Brown M.J.F., Baer B. \& Schmid-Hempel P. 2001: Males of social insects can prevent queens from multiple mating. Proc. R. Soc. Lond. (B) 268: 1449-1454.

Schmid-Hempel R. \& Schmid-Hempel P. 2000: Female mating frequencies in Bombus spp. from Central Europe. Insectes Soc. 47: 36-41.

Schoeters E. \& Billen J. 2000: The importance of the spermathecal duct in bumblebees. J. Insect Physiol. 46: 1303-1312.

Simmons L.W. 2001: Sperm Competition and its Evolutionary Consequences in the Insects. Monographs in Behaviour and Ecology. Princeton University Press, Princeton, NJ, 448 pp. 
Tasei J.N., Moinard C., Moreau L., Himpens B. \& Guyonnaud S. 1998: Relationship between aging, mating and sperm production in captive Bombus terrestris. J. Apicult. Res. 37: 107-113.

Trivers R.L. \& Hare H. 1976: Haplodiploidy and evolution of social insects. Science 191: 249-263.

Weber N.A. 1972: Gardening Ants: The Attines. American Philosophical Society, Philadelphia, $146 \mathrm{pp}$.

Widmer A. \& Schmid-Hempel P. 1999: The population genetic structure of a large temperate pollinator species, Bombus pas- cuorum (Scopoli) (Hymenoptera: Apidae). Mol. Ecol. 8: 387-398.

WOLFNER M.F. 2002: The gifts that keep on giving: physiological functions and evolutionary dynamics of male seminal proteins in Drosophila. Heredity 88: 85-93.

Zен J.A. \& ZeH D.W. 2003: Toward a new sexual selection paradigm: Polyandry, conflict and incompatibility. Ethology 109: 929-950.

Received June 5, 2008; revised and accepted August 15, 2008 